\title{
An Atomic Renaissance For Pulsed Field Ion Microscopy
}

Shyam Katnagallu ${ }^{1}$, Isabelle Mouton ${ }^{1}$, Felipe Oliveira ${ }^{1}$, Baptiste Gault ${ }^{1}$, Dierk Raabe ${ }^{1}$, Leigh T. Stephenson $^{1}$

1. Max Planck Institute für Eisenforschung, Düsseldorf, Germany

Corresponding author: 1.stephenson@mpie.de

Field ion microscopy naturally led to the development of atom probe tomography via the late-1960s transitional form of atom probe FIM instruments [1] which combined FIM imaging with energy or timeof-flight mass spectrometry through a small probe hole in a phosphorescent imaging screen (hence atom "probe"). Despite being related through this hybrid technique, most modern instruments are optimally designed for either FIM imaging or APT reconstructions. For some materials, FIM can supply atomically resolved images and modern experiments have furnished 3D-FIM reconstructions that can visualize crystallographic defects, precipitate morphologies and dopant distributions [2,3]. APT is more widely applicable to the material sciences and has been exploited many nanostructural studies [4]. An interesting question is whether a hybrid technique using modern instrumentation can provide information that is otherwise inaccessible to either pure technique. That is the basis of our investigation described here and the answer is yes with some results described here.

We used two Cameca Local Electrode Atom Probes (LEAP 3000HR/5000XS) with commerciallyavailable FIM accessories. Both are fitted with high purity gas bottles (He/Ne/Ar) and a mixing chamber with a direct inlet into the LEAP analysis chamber. Instead of the traditional phosphor screen, the LEAP's delay line detector (coupled with a multichannel plate) is used for realizing an electronic FIM (eFIM) capability. Once installed, two obvious software limitations prevented more widespread research using eFIM. The supplied eFIM control software module integrates the detected ions into a FIM image and does not record individual hit positions. Additionally, this module allows no voltage/laser pulsing and offers only a manual control upon the standing voltage, making it difficult to acquire FIM imaging of progressive field evaporation for 3DFIM reconstructions. Substituting the eFIM module with the standard APT control module under FIM experimental conditions was a straightforward experiment, trialed first by a start-up in University of Sydney in 2015 [5] with related research commencing in late 2017 at the Max Planck Institute for Iron Research. Compared to the eFIM module, this approach gave more experiment control for FIM imaging but, as for an APT experiment, furnished the imaging with much more data for each detected ion, i.e. time-of-flights, projected position, and other supplementary information [6]. This is demonstrated in Figure 1.

Here, we consider three main aspects regarding this hybrid technique - here called "time-of-flight enabled field ion microscopy". Firstly, we consider the set of materials science problems for which using this hybrid technique may be of some benefit. Then we outline various experimental protocols to separate field evaporated signals from the large field ionization background signal, employing various data mining strategies for this discrimination. Lastly, we consider the outlook for such investigations, what experimental challenges need addressing and whether this heralds a field ion microscopy revival.

References: 
[1] EW Müller, JA Panitz, SB McLane, “The Atom-Probe Field Ion Microscope”, Review of Scientific Instruments 39 (1968) p. 83.

[2] F Vurpillot, M Gilbert, B Deconihout, "Towards the three-dimensional field ion microscope", Surface and Interface Analysis 39 (2007) p. 273.

[3] S Koelling et al., "Direct imaging of 3D atomic-scale dopant-defect clustering processes in ionimplanted silicon", Nano Letters 13 (2013) p. 2458.

[4] DN Seidman, "Three-Dimensional Atom-Probe Tomography: Advances and Applications", Annual Review of Materials Research 37 (2007) 127-158

[5] Åtomnaut, http://www.atomnaut.com/ (accessed February 14, 2019).

[6] B Gault, MP Moody, JM Cairney, SP Ringer, “Atom Probe Microscopy”, Springer New York (2012)

FIM

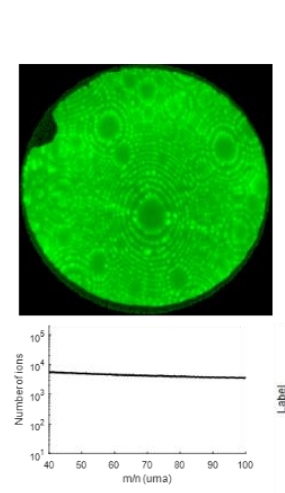

Pulsed "tof-FIM"

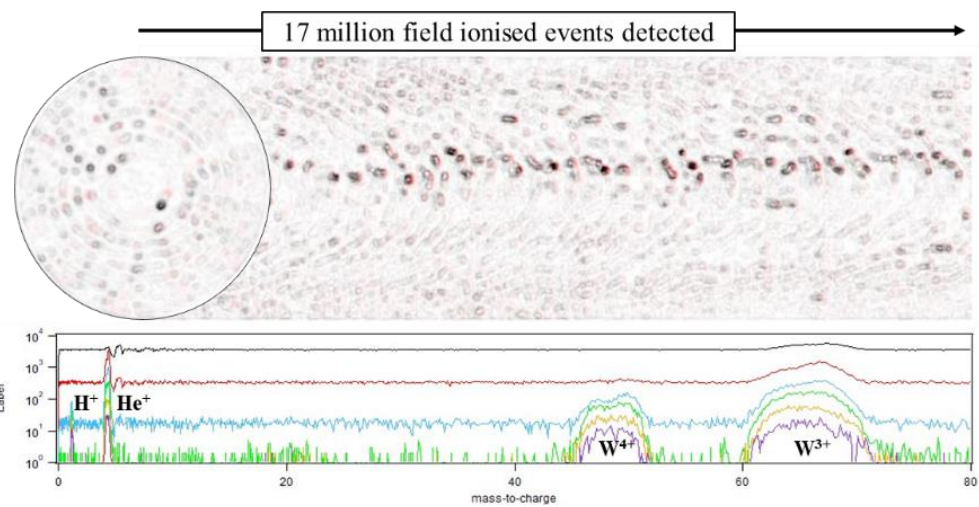

APT
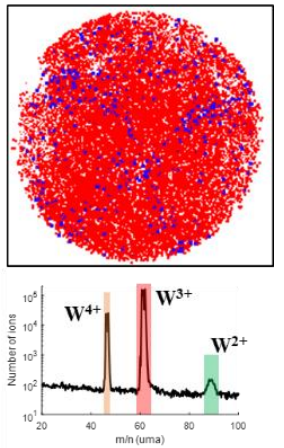

Figure 1. For a tungsten tip we can contrast three experiments. (Left) A conventional FIM experiment without pulsing demonstrates a time-of-flight mass-to-charge spectrum with a flat imaging gas background. (Right) A standard APT experiment employing voltage pulsing within a clean UHV chamber realizing a clean time-of-flight chemical analysis but without the spatial accuracy of FIM. (Middle) Between the two conventional experimental paradigms, pulsed "tof-FIM" employs pulsing, allowing for both high spatial precision and mass spectrometry. Discrimination between the field ionised background and the field evaporated ions can be achieved in examining the multiple mass-to-charge signals. 